\title{
Monodisperse self-assembly in a model with protein-like interactions
}

\author{
Alex W. Wilber, ${ }^{1}$ Jonathan P. K. Doye,,${ }^{1,}$ Ard A. Louis, ${ }^{2}$ and Anna C. F. Lewis ${ }^{1}$ \\ ${ }^{1}$ Physical and Theoretical Chemistry Laboratory, Department of Chemistry, \\ University of Oxford, South Parks Road, Oxford, OX1 3QZ, United Kingdom \\ ${ }^{2}$ Rudolf Peierls Centre for Theoretical Physics, University of Oxford, \\ 1 Keble Road, Oxford, OX1 3NP, United Kingdom
}

(Dated: November 1, 2018)

\begin{abstract}
We study the self-assembly behaviour of patchy particles with 'protein-like' interactions that can be considered as a minimal model for the assembly of viral capsids and other shell-like protein complexes. We thoroughly explore the thermodynamics and dynamics of self assembly as a function of the parameters of the model and find robust assembly of all target structures considered. Optimal assembly occurs in the region of parameter space where a free energy barrier regulates the rate of nucleation, thus preventing the premature exhaustion of the supply of monomers that can lead to the formation of incomplete shells. The interactions also need to be specific enough to prevent the assembly of malformed shells, but whilst maintaining kinetic accessibility. Free-energy landscapes computed for our model have a funnel-like topography guiding the system to form the target structure, and show that the torsional component of the interparticle interactions prevents the formation of disordered aggregates that would otherwise act as kinetic traps.
\end{abstract}

PACS numbers: 81.16.Dn,87.15.ak,87.15.km,81.16.Fg

\section{INTRODUCTION}

The self-assembly of simple building blocks into larger, ordered structures is a ubiquitous process in biology, and holds great promise for applications in materials science and nanotechnology ${ }^{12}$ In particular, proteins are the building blocks for a vast array of biological structures, including capsules, fibres, tubes, sheets, channels and motors. Here, we restrict our interest to those examples where the self-assembly is both one-component and monodisperse, i.e. the structures formed are of a specific size and made up of multiple copies of the same protein. The archetypal examples of this type are icosahedral virus capsids, which are designed to encapsulate the genomic material of the virus. The capsid proteins are produced in large quantities inside host cells, and need to assemble correctly and spontaneously on a biological time scale in order for the virus to propagate. While many capsid assembly processes rely on interactions between the capsid proteins and scaffolding proteins or nu-

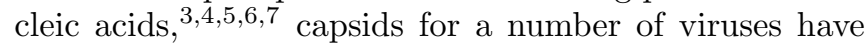
been shown to assemble in vitro in the absence of these molecules. $8|9| 10|11| 12$

There are also a significant number of non-viral proteins that form shell-like homomeric complexes with high symmetry! ${ }^{13|14| 15}$ For example, ferritin is a complex that is made up of 24 sub-units with octahedral symmetry that stores iron inside its central cavity, 16 drolipoyl acetyltransferase can form a 60-unit dodecahedral complex that is at the core of the pyruvate dehydrogenase multienzyme complex ${ }^{17}$ However, the selfassembly behaviour of such complexes has been much less studied than for virus capsids.

Clearly, establishing a good understanding of the nature of the self-assembly process of protein complexes and virus capsids, as well as being of fundamental inter- est, will be of value to many potential biomedical and bionanotechnological applications, e.g. in the design of drugs which interfere with capsid assembly $18|19| 20$ or the use of capsids as vehicles for the delivery of drugs or other agents. $21 \mid 22$ Finally, an understanding of the features that enable such robust assembly in these protein systems may be very valuable in designing synthetic selfassembling systems. $23 \mid 24$

There is now a considerable body of work studying the assembly of virus capsids, both experimentally $6|7| 11|12| 25|26| 27|28| 29|30| 31 \quad$ and theoretically. $32|33| 34|35| 36|37| 38|39| 40|41| 42|43| 44|45| 46|47| 48|49| 50|51| 52$ The transient nature of the intermediates present a particular challenge to the experiments. By contrast, characterizing species with short lift-times is much less of an obstacle for simulation and modelling, and so they can play an important and complementary role by illuminating the mechanisms of assembly. Instead, the problem for simulations is the wide range of time and length scales associated with capsid assembly. For example, although all-atom simulations of small viruses are now possible,, 53 the time scales associated with self-assembly are far beyond what is currently feasible. Therefore, it is necessary to use much simpler coarsegrained models, the hope being that if self assembly has general rules as to what conditions and subunit designs lead to successful assembly, these should be accessible through such models.

In this vein, a number of computational approaches have been directed towards capsid assembly. Kinetic models consider the populations of particular clusters and model cluster growth by assigning rate constants to combination/breakup events, using either a differential equation $\stackrel{32 \mid 36}{ }$ or a discrete event approach. ${ }^{4142}$ However, these models do not include any information about the spatial positions of subunits, and only consider a lim- 
ited set of assembly pathways. Simulations do not suffer from these restrictions, but it is more challenging to generate good statistics and thoroughly analyse the parameter space. Indeed, it is only relatively recently that models with fully reversible dynamics have been studied. The simulations can be grouped into two types depending on whether the protein subunits are modelled as anisotropic objects consisting of a rigid structure of smaller spheres $34 \mid 35 / 46 / 47 / 48$ or as spherical particles with anisotropic 'patchy' interactions. ${ }^{43}$ The latter approach is typically computationally less intense, and is the one we use here.

Previously, model to study the assembly of icosahedral clusters, and in the accompanying paper this study is extended to the remainder of the Platonic solids. ${ }^{55}$ However, in these studies the model included no constraints on the torsional (dihedral) angle between interacting particles. While this is likely to be appropriate for synthetic 'patchy' colloids and nanoparticles, 2456 it does not represent proteinprotein interactions well since the complex structure of the interfaces between the proteins will tend to restrict rotation. Such torsional constraints are also relevant to recently created 3- and 5-armed DNA building blocks that can assemble into tetrahedra, dodecahedra, truncated icosahedra,,$[57$ cubes 58 and icosahedra,, 59 and where the relative orientation of these units is controlled by the number of turns of the DNA double helix along each arm.

Here we consider the effects of adding a torsional component to the interparticle potential of this minimal model, and proceed to map out the behaviour of our systems over a wide region of parameter space. We examine the dynamics and mechanisms of assembly, and consider the behaviour of the system in regions in which assembly is not successful in order to understand those processes which compete with successful assembly. This work has a number of distinctives compared to previous simulation studies of viral assembly. Firstly, we do not just consider the assembly of an icosahedral target that is equivalent to a $T=1$ capsid, but more generally consider structures with other symmetries that are also relevant to non-viral protein complexes. Secondly, the model is simpler than those previously studied, thus allowing us to survey the behaviour of the model very comprehensively. In particular, we are able to connect the dynamics to the underlying thermodynamics of the model, and obtain detailed pictures of the free energy landscapes governing assembly. Finally, comparison to the non-torsional model considered previously, allows us to understand what features of the behaviour arise because of this component in the interparticle interactions, and such information will be particularly relevant for those trying to design synthetic particles that are able to self-assemble.

\section{METHODS}

\section{A. Model}

The model system we use is a slightly modified version of that used previously $\sqrt[5460161]{ }$ and in the accompanying paper ${ }^{[55}$ It has also recently been used to study the assembly of tetrameric protein complexes. ${ }^{62}$ The model consists of spherical particles with a number of sticky patches, which are defined by patch vectors. The interaction potential is pairwise and is based on the LennardJones potential,

$$
V_{\mathrm{LJ}}(r)=4 \epsilon\left[\left(\frac{\sigma_{\mathrm{LJ}}}{r}\right)^{12}-\left(\frac{\sigma_{\mathrm{LJ}}}{r}\right)^{6}\right],
$$

but the attraction is modulated by a pair of orientationally dependent terms, $V_{\text {ang }}$ and $V_{\text {tor }}$. $V_{\text {ang }}$ is a function of how directly the two patches are pointing at one another, while the additional factor $V_{\text {tor }}$ is a function of the torsional angle between the two particles, i.e. it varies as one of the particles is rotated about the vector connecting the two particles. Thus, the complete potential is

$$
\begin{aligned}
& V_{i j}\left(\mathbf{r}_{i j}, \boldsymbol{\Omega}_{i}, \boldsymbol{\Omega}_{j}\right)= \\
& \quad \begin{cases}V_{\mathrm{LJ}}\left(r_{i j}\right) \\
V_{\mathrm{LJ}}\left(r_{i j}\right) V_{\mathrm{ang}}\left(\hat{\mathbf{r}}_{i j}, \boldsymbol{\Omega}_{i}, \boldsymbol{\Omega}_{j}\right) V_{\mathrm{tor}}\left(\hat{\mathbf{r}}_{i j}, \boldsymbol{\Omega}_{i}, \boldsymbol{\Omega}_{j}\right) & r \geq \sigma_{\mathrm{LJ}},\end{cases}
\end{aligned}
$$

where $\boldsymbol{\Omega}_{i}$ is the orientation of particle $i$. For any pair of particles only the pair of patches that maximizes $V_{\text {ang }} V_{\text {tor }}$ are considered to interact. $V_{\text {ang }}$ has the form:

$$
\begin{aligned}
V_{\text {ang }}\left(\hat{\mathbf{r}}_{i j}, \boldsymbol{\Omega}_{i}, \boldsymbol{\Omega}_{j}\right) & =G_{i j}\left(\hat{\mathbf{r}}_{i j}, \boldsymbol{\Omega}_{i}\right) G_{j i}\left(\hat{\mathbf{r}}_{j i}, \boldsymbol{\Omega}_{j}\right) \\
G_{i j}\left(\hat{\mathbf{r}}_{i j}, \boldsymbol{\Omega}_{i}\right) & =\exp \left(-\frac{\theta_{k i j}^{2}}{2 \sigma_{\text {ang }}^{2}}\right)
\end{aligned}
$$

where $\sigma_{\text {ang }}$ is a measure of the width of the Gaussian, $\theta_{k i j}$ is the angle between patch vector $k$ on particle $i$ and the interparticle vector $\mathbf{r}_{i j}$.

In the calculation of $V_{\text {tor }}$, an additional reference vector on each particle needs to be defined. We have chosen this reference vector in each case to lie on the symmetry axis of the particle. $V_{\text {tor }}$ is a maximum when the projections of the two reference vectors onto the plane perpendicular to the interparticle vector lie parallel. Specifically, if $\phi$ is the angle between the projections, then

$$
V_{\mathrm{tor}}=\exp \left(-\frac{\phi^{2}}{2 \sigma_{\text {tor }}^{2}}\right)
$$

where $\sigma_{\text {tor }}$ is a measure of the width of the Gaussian. The effect of the inclusion of $V_{\text {tor }}$ in the potential is to penalise twisting around the interparticle vector, with smaller values of $\sigma_{\text {tor }}$ giving a stronger constraint. In order to reduce the number of parameters to consider and because we consider it physically reasonable that $\sigma_{\text {tor }}$ and $\sigma_{\text {ang }}$ are coupled, we set $\sigma_{\text {tor }}=2 \sigma_{\text {ang }}$ throughout this paper, so that the specificity of interactions is given only 
in terms of $\sigma_{\text {ang. }}$ Our results are relatively insensitive to the precise value of the ratio of these parameters.

The patch vectors are chosen such that they point directly at the neighbouring particles in the target structure. This choice, along with that of the reference vector above, ensures that the target structures are lowest in energy for sufficiently specific patches. The target clusters that we consider are the five Platonic solids. Thus, each particle has internal $C_{n}$ symmetry because of the presence of $n$ equivalent and regularly-arranged patches. Therefore, when comparing to virus capsids and other large homomeric protein complexes, the particles do not represent individual proteins, which have no symmetry, but cyclic protein complexes that can further assemble into larger complexes. For example, the icosahedronforming particles could be considered to represent the pentameric capsomers often posited as intermediates in viral capsid assembly ${ }^{26 / 29}$ and the complete icosahedron a $T=1$ capsid. Similarly, the dodecahedron-forming particles could represent dihydrolipoyl acetyltransferase trimers ${ }^{17}$ In this context, we are modelling the second stage of a hierarchical self-assembly process. 63

For computational efficiency we use a cut-and-shifted version of the potential with a cutoff distance of $3 \sigma_{\mathrm{LJ}}$, and also shift the crossover distance in Eq. 2 so that it occurs when the cut-and-shifted potential passes through zero.

\section{B. Simulation}

All our simulations are based on Metropolis Monte Carlo simulations in the canonical ensemble. We restrict the translational and rotational moves to be local, so that the particle motion mimics the diffusive behaviour of proteins and nanoparticles in solution. Where equilibrium statistics are needed we make use of the umbrella sampling technique, applying an order parameter dependent bias to facilitate the crossing of free energy barriers. For more details see the accompanying paper!54

A particle number density of $0.15 \sigma_{\mathrm{LJ}}^{-3}$ is used for all simulations. This represents a significantly higher concentration than would normally be considered in in vitro studies involving proteins. Because the time scales accessible to simulation are far shorter than those available to experiment, it is necessary to use higher concentrations in order to observe assembly. For our original model without torsional constraints we found that for concentrations in the range $0.025-0.4 \sigma_{\mathrm{LJ}}^{-3}$ the final yields only show a weak dependence of on concentration.

\section{RESULTS}

In order to map the behaviour of our systems over a wide region of parameter space, we performed large arrays of simulations with varying values of the patch width $\sigma_{\text {ang }}$ and the temperature $T$, for each of the Platonic
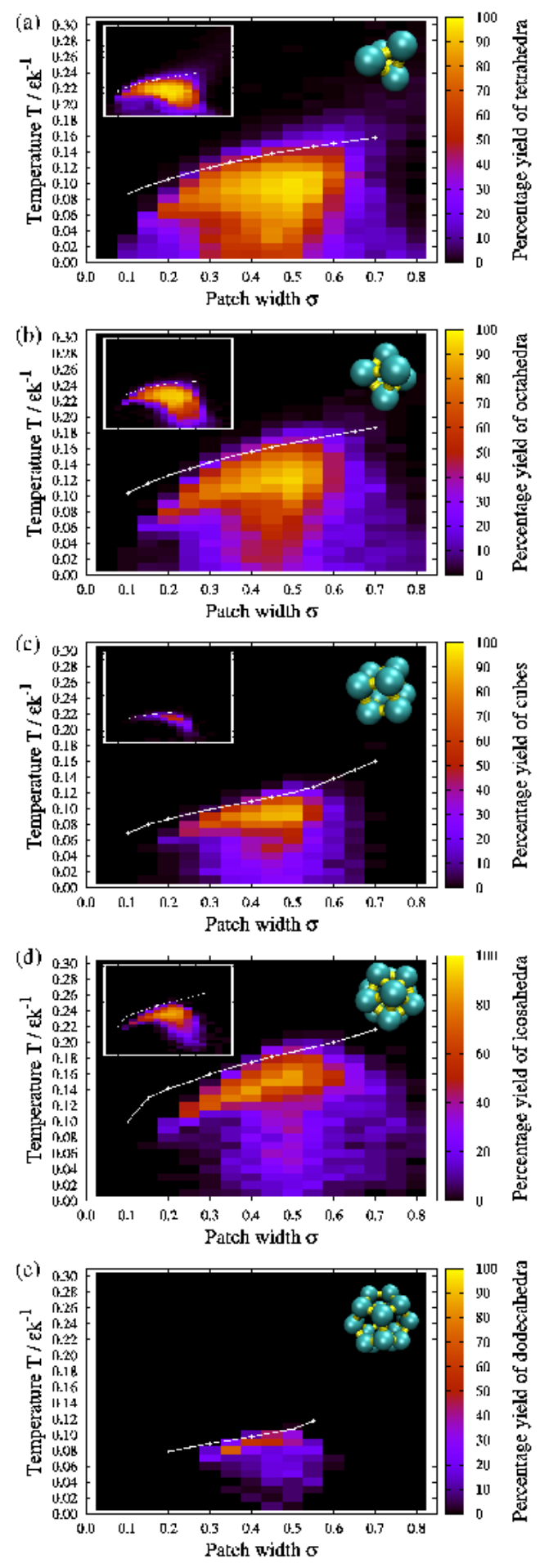

FIG. 1: (Colour Online) The percentage yield of target clusters formed after $80000 \mathrm{MC}$ cycles as a function of the patch width $\sigma_{\text {ang }}$ (measured in radians) and temperature at a number density of $0.15 \sigma_{\mathrm{LJ}}^{-3}$ for systems of 1200 particles designed to form (a) tetrahedra, (b) octahedra, (c) cubes, (d) icosahedra and (e) dodecahedra. The insets show the equivalent plots for simulations without torsional constraints, where the axes span the same parameter ranges as the main plots. No inset is included for dodecahedra since without torsional constraints there was no conditions under which dodecahedra assembled. The images in the top right of each plot show the relevant target structure. 
solids. The results are shown in Figs. 1 and 2 Note that, although we will typically talk about the dependence of the behaviour on temperature, we could equivalent have used the interaction strength, where decreasing temperature corresponds to increasing interaction strength. In Fig. 1. which gives the yields of successfully assembled clusters, a region of high yield is visible for each target structure at moderate values of temperature and patch width, where the target is thermodynamically stable and kinetically accessible. Moving away from this region, yields fall away as competing processes become dominant.

The high temperature region corresponds to a gas of monomers and small clusters. Above a certain temperature, which we term $T_{c}$, the target clusters cease to be stable with respect to this high entropy gas. This temperature is a strong function of the patch width $\sigma_{\text {ang }}$ since wider patches lead to higher entropies for the target clusters, arising from internal vibrations. Fig. 2, which shows the average cluster sizes achieved as a function of patch width and temperature, clearly shows the extent of the monomer gas region.

In the early stages of the simulations below $T_{c}$, the particles tend to rapidly assemble into curved shell-like structures. The inclusion of torsional constraints in the potential favours a certain curvature in the structures formed, such that even erroneous structures are typically still hollow shells of roughly the correct size and shape. In particular the constraints tend to enforce convexity on the structures, greatly restricting the range of possible structures which can compete with the target structure.

At high values of the patch width $\sigma_{\text {ang }}$, the interactions between particles become less specific. Low energies can still be obtained by significantly distorted clusters, and so with larger $\sigma_{\text {ang }}$ increasingly malformed clusters are observed, until correctly formed clusters become a rarity. These malformed clusters are somewhat similar to the monster particles which have been observed experimentally in Turnip Crinkle Virus $\stackrel{25}{25}$ and in simulations by Nguyen et al. . ${ }^{46}$ However, the nature of our interaction potential limits the range of structures which can be formed, so that the fused-shell structures seen in these papers are not seen. Instead we find convex structures similar to those found in more recent work by Nguyen and Brooks ${ }^{47}$ At still higher values of $\sigma_{\text {ang }}$ the patches are sufficiently wide that they lose their specificity, and instead of forming hollow shells, the system forms large, roughly spherical liquid-like droplets.

At low temperatures more complicated behaviour is observed, with generally lower yields but some successful assembly even at very low temperatures for moderate values of $\sigma_{\text {ang }}$. The low yields arise because rapid nucleation leads to the removal of almost all the monomers from the system by the growing clusters, leaving many clusters half-formed and unable to grow further. This phenomenon of monomer starvation is a recurring theme of virus assembly, and has been reported in a number of previous experimental studies, $\frac{6 / 7}{6}$ as well as being pre-
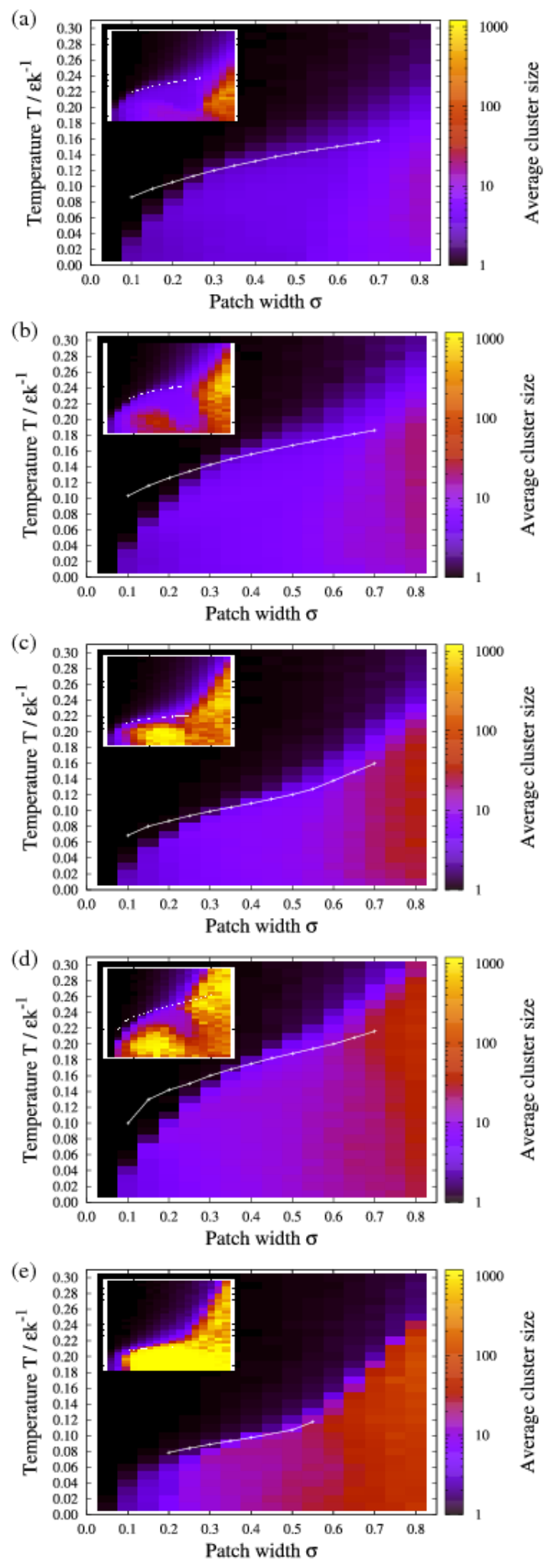

FIG. 2: (Colour Online) The mean cluster size (averaged over particles) of systems designed to form (a) tetrahedra, (b) octahedra, (c) cubes, (d) icosahedra and (e) dodecahedra, for the same simulations as Fig. 1. The insets show the equivalent plots for simulations without torsional constraints, where the axes span the same parameter ranges as the main plots. 

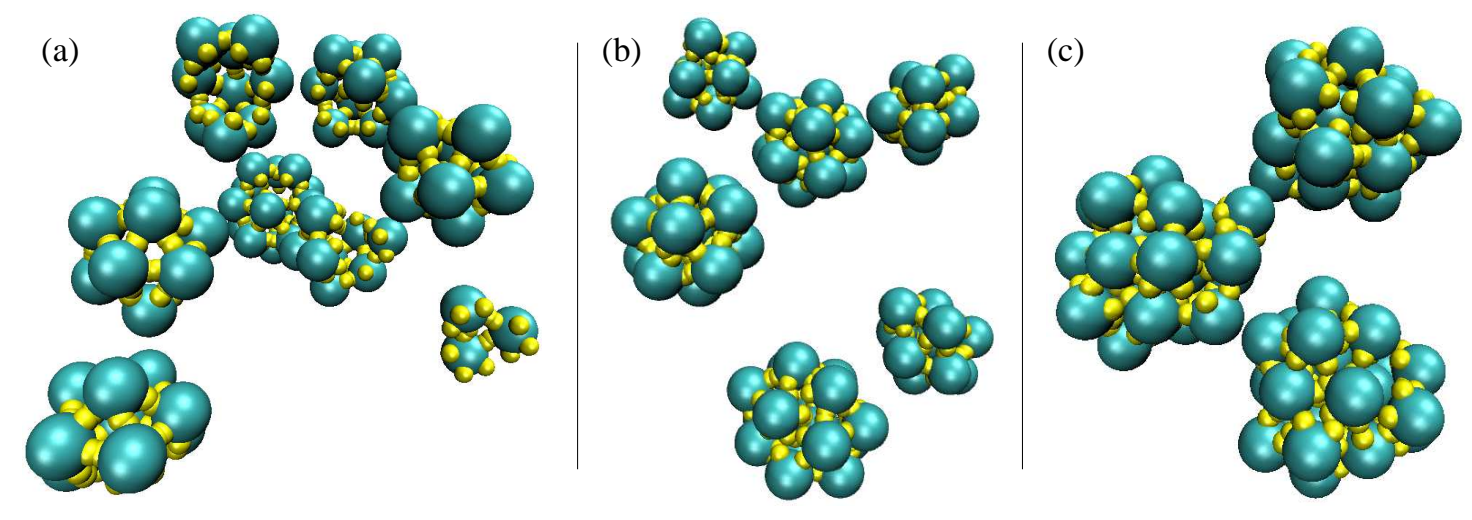

FIG. 3: (Colour Online) Snapshots of typical configurations of 72 icosahedron-forming particles. (a) $T=0.08, \sigma_{\text {ang }}=0.35$, showing monomer starvation, (b) $T=0.08, \sigma_{\text {ang }}=0.6$, showing misformed clusters, and (c) $T=0.16, \sigma_{\text {ang }}=0.75$, showing liquid droplets.

dicted by kinetic model ${ }^{32 / 41}$ and simulations. $\frac{34 \sqrt[35 / 43 / 46]{3}}{3}$ We shall return later to consider the reason for the recovery in yield at moderate patch widths.

Fig. 3 shows typical configurations for small systems in different regions of parameter space for the icosahedronforming particles. Fig. 3(a) shows a system displaying monomer starvation. Six partially formed clusters are visible, as well as one trimer and one 14-particle cluster which is not easily able to rearrange because of the low temperature and patch width. This system is unlikely to form any correctly formed clusters within accessible timescales. Fig. 3(b) shows a system at higher patch widths, such that misformed clusters are relatively low in energy and are frequently observed. Two correctly formed icosahedra are visible, along with a number of misformed clusters. Finally, Fig. 3(c) shows liquid droplets formed at very high $\sigma_{\text {ang. }}$. Unlike the misformed clusters seen in Fig. 3(b), these droplets are not hollow, and the particles are mobile within the droplets.

The general form of the behaviour of the systems is to first order independent of the target structure. The plots for each of the targets in Figs. 1 and 2 have essentially the same form in each case. However, some differences are also observed. Most strikingly, the temperature delineating the transition from target clusters to a monomer gas, $T_{c}$, varies considerably. In general $T_{c}$ is greater for smaller clusters (since there is a lower entropic cost for the formation of these clusters), and also greater for higher numbers of patches per particle (since the clusters are more energetically favoured). These effects lead to fairly similar values of $T_{c}$ for the tetrahedron, octahedron and icosahedron, where the differences from the two effects largely cancel, and lower values for the cube and the dodecahedron.

The insets in Figs. 1 and 2 show the results for equivalent systems without torsional constraints. The most striking differences are visible in Fig. 2, where the insets show that in the absence of torsional constraints, extremely large clusters are formed over wide ranges of parameter space. These large clusters correspond to dis- ordered kinetic (at low $T$ ) or thermodynamic (at high $\left.\sigma_{\text {ang }}\right)$ aggregates. Torsional constraints prevent the formation of these large and disordered aggregates by enforcing convexity, the only exception being at the largest values of $\sigma_{\text {ang }}$ in Fig. 2 that are well away from the region of successful assembly.

For systems without torsional constraints, the competition between aggregation and self-assembly can severely limit the yield of the target structure $\frac{[55}{5}$ The effects of this competition are particularly noticeable for cubes, where assembly is limited to a small region, and dodecahedra, where assembly does not occur at all. For these two targets the yields dramatically increase when torsional constraints are included because the competing aggregates cannot form.

For the targets that readily assemble with and without torsional constraints, there is one region in which the yield is clearly decreased by the inclusion of torsional constraints. At patch widths slightly higher and temperatures slightly lower than the optimum, the systems without torsional constraints assemble by a "budding mechanism" ${ }^{54}$ In this mechanism the particles first coalesce into disordered aggregates, which then rearrange and bud off completed clusters. Since torsional constraints prevent the formation of large aggregates, they have the effect of inhibiting this indirect assembly mechanism.

These effects are clearly visible in the different shapes of the regions of successful assembly with and without torsional constraints in Fig. 1. The "lobe" of successful assembly due to budding is missing in the simulations with torsional constraints, while a new region of partially successful assembly arises at low temperature and moderate patch widths, in part due to the lack of competition with large kinetic aggregates.

Assembly in systems with torsional constraints proceeds exclusively by the stepwise addition of monomers and small clusters onto growing clusters. Fig. 4 shows the average cluster size as a function of time at different temperatures, with and without torsional constraints. While 


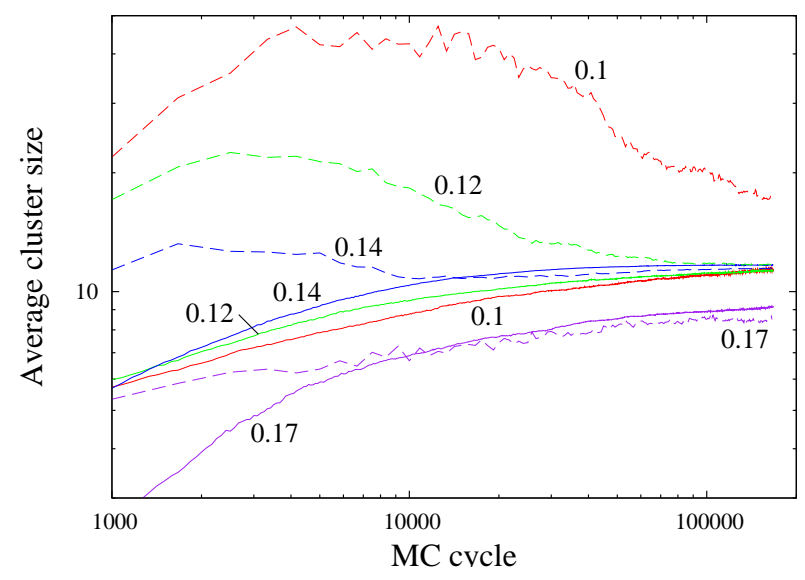

FIG. 4: (Colour Online) The mean cluster size (sampled over particles) as a function of number of MC cycles at $\sigma_{\text {ang }}=0.45$ and at different temperatures (as labeled) for a system of 1200 icosahedron-forming particles. The dashed lines show the equivalent results for systems without torsional constraints. Each line is an average over ten simulations.

in the absence of torsional constraints the average cluster size sometimes approaches the target size from above (indicating that assembly proceeds by the budding mechanism), the average sizes for the simulations which include torsional constraints all approach the target size from below, consistent with a stepwise growth mechanism.

Figure 5 provides a detailed picture of the dynamics by showing the numbers of particles in clusters of a given size as a function of time, under four different sets of conditions for the example of the icosahedron. Under optimal assembly conditions (Fig. 5(a)), there is only a small population of intermediate-sized clusters and as time progresses the population of monomers falls off slowly as more complete icosahedra are formed. That the population of intermediate-sized clusters is always less than monomers or icosahedra (after an initial lag time required for icosahedra to start forming) is indicative of a significant free energy barrier for the formation of the target structure. As has been noted previously, $6 / 7] 32[41$ relatively slow nucleation is a prerequisite for successful capsid assembly. When few nuclei are formed, the population of monomers remains high for longer allowing the majority of the nuclei to grow to completion.

At lower temperatures, such as in Fig. 5(b), the effect of monomer starvation is evident. Under these conditions, the formation and growth of cluster is all downhill in free energy. The consequent rapid nucleation of clusters leads to a rapid decrease in the population of monomers, and many of the partially formed clusters are unable to grow to completion. At this point assembly is effectively stalled, and can only proceed through a slow process of breakup of the existing clusters. This is the reason for the low yields observed at low values of $T$ and $\sigma_{\text {ang }}$.

For these combination of reasons, optimal assembly oc- curs fairly close to $T_{c}$. This result is consistent with Zlotnick's assertion that "weak" bonds are sufficient and indeed optimal for efficient assembly of viruses 3764 (but of course while still being strong enough that the capsids are stable) 67

Although the phenomenon of monomer starvation is found at low temperature regardless of $\sigma_{\text {ang }}$, yields are found to recover for moderate patch widths. This recovery is observed in Fig. 5(c) and is due to an interesting mechanism of combination and rearrangement of clusters. Pairs of partly formed clusters occasionally collide and stick together. Most often the two parts will not fit perfectly to form a complete cluster, and so a process of rearrangement then occurs. If excess particles are present, they may often be ejected from the rearranging cluster. Even closed shells are able to take advantage of this mechanism if they contain too few particles. If a small cluster approaches a strained region of a shell it may be able to insert itself to form a complete target cluster; again, any unneeded particles may be ejected. These events are readily visible in movies of our simulations. As an example of a typical event, a ten-particle closed cluster was observed to encounter a three-particle triangle. The triangle became loosely bound to the outside of the closed shell, then over time the particles in the shell moved apart such that two particles from the triangle were able to incorporate into the shell to form a perfect icosahedron. The bonds with the remaining particle were broken, and it diffused away.

These processes depend on $\sigma_{\text {ang }}$ taking at least a moderate value for several reasons. Firstly, collisions between partly formed clusters are much more likely to result in binding interactions when the patches are wider. Secondly, insertion of small clusters into closed shells only becomes possible when the patches are sufficiently wide that both the inserting fragment can become attached to the outside of the shell, despite the highly suboptimal angles between the respective patches, and the distortion required for insertion is feasible. Most importantly, the energy barriers for rearrangement are greatly decreased with wide patches, since the intermediate states can be stabilised by interactions between poorly aligned patches. These mechanisms of combination and rearrangement lead, for moderate values of $\sigma_{\mathrm{ang}}$, to reasonable yields at long times even at very low temperatures.

For even higher values of $\sigma_{\text {ang }}$, yields fall off once more, and the kinetics in this regime are illustrated in Fig. 5(d). While the mechanisms of combination and rearrangement remain effective (hence the number of small intermediates again falls off with time), the system is no longer so strongly driven to form correctly assembled clusters, since mildly misformed clusters will have similar energies per particle to correctly formed clusters. Excess particles will only rarely be ejected, and many of the clusters formed are oversized.

Fig. 6 provides an alternative perspective on many of the effects discussed above. It shows the yields as a function of temperature and time for each of the shapes, at 

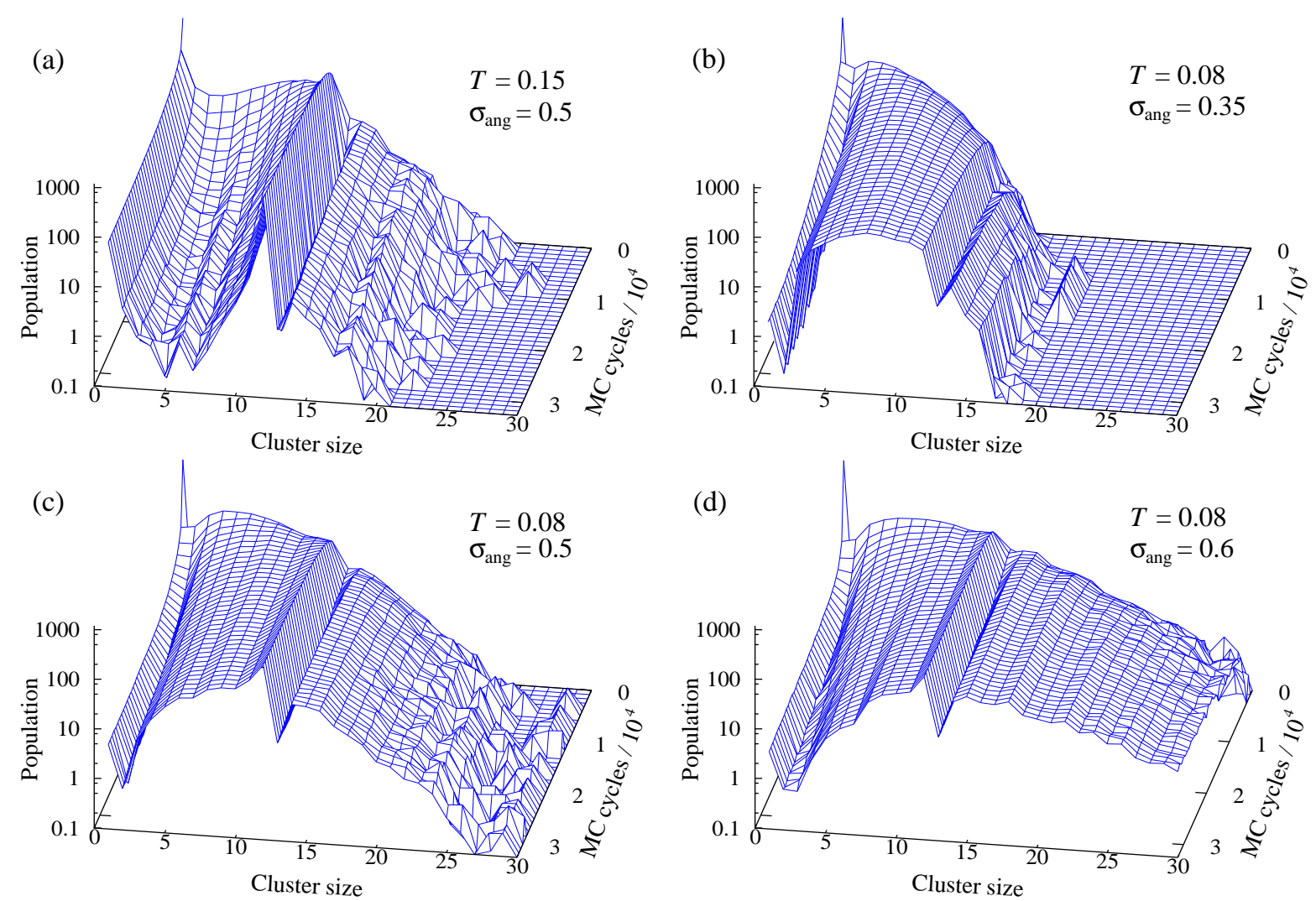

FIG. 5: (Colour Online) The populations of particles in clusters of different sizes as a function of number of MC cycles for systems of 1200 icosahedron-forming particles, averaged over 100 repetitions. The temperatures and patch widths are as labelled, such that (a) is at the optimal conditions for assembly, (b) is in the region of monomer starvation, (c) shows recovery at moderate patch widths, and (d) shows a loss of specificity at high patch widths. A log scale is used so that the very small populations of intermediates are discernible. States with zero population have been set to a minimum value of 0.1 to aid in visualisation.

the optimal value of $\sigma_{\text {ang }}$ in each case. A number of effects are visible. Considering first the plot for icosahedra, Fig. 6(d), a sharp cut-off in the early yields is seen at around $T=0.13$, corresponding to the onset of monomer starvation. Yields below this cutoff recover at longer times, as a result of the combination and rearrangement of clusters. Similar behaviour is observed for the other shapes, although the sharpness of the monomerstarvation cutoff decreases for the smaller shapes, since monomer starvation affects smaller targets less seriously because there are a smaller number of intermediate states in which to get trapped. Furthermore, recovery is also generally easier for the smaller targets, as less rearrangement is likely to be required. The virtual absence of any recovery in Fig. 6(e) is primarily because optimal assembly for the dodecahedron occurs at a lower value of $\sigma_{\text {ang }}$; there is some evidence of some recovery at larger values of $\sigma_{\text {ang }}$ in Fig. 1 1 (e).

Fig. 7 shows the yields for each target structure as a function of time. The kinetics is seen to be sigmoidal, with an initial lag phase during which intermediates of increasing sizes build up in turn before the first complete clusters are formed. This is a ubiquitous feature in simulations of virus assembly, and indeed predicted for all multistep reactions ${ }^{65}$ For larger targets the lag phase is longer, since the reaction has to progress through a larger number of intermediates. In all cases the yield approaches $100 \%$ at long times.

The successful formation of dodecahedra is a particularly notable consequence of introducing torsional constraints. In the absence of torsional constraints the assembly of dodecahedra is entirely prevented by competition with disordered aggregates, which are more thermodynamically stable at high temperatures, and which rearrange only extremely slowly at lower temperatures (for more details see the accompanying paper.55) However, since the formation of aggregates is largely prevented by the inclusion of torsional constraints the formation of dodecahedra becomes possible, and indeed approaches $100 \%$ yield at long times. Indeed, Fig. 7 presents a picture of the time scale for assembly increasing monotonically with target size with no obviously anomalous behaviour due to particular geometric features of a target.

We can see the effect of this lack of competition with aggregation in the thermodynamics of the assembly process. We consider first Fig. 8, which shows the heat ca- 

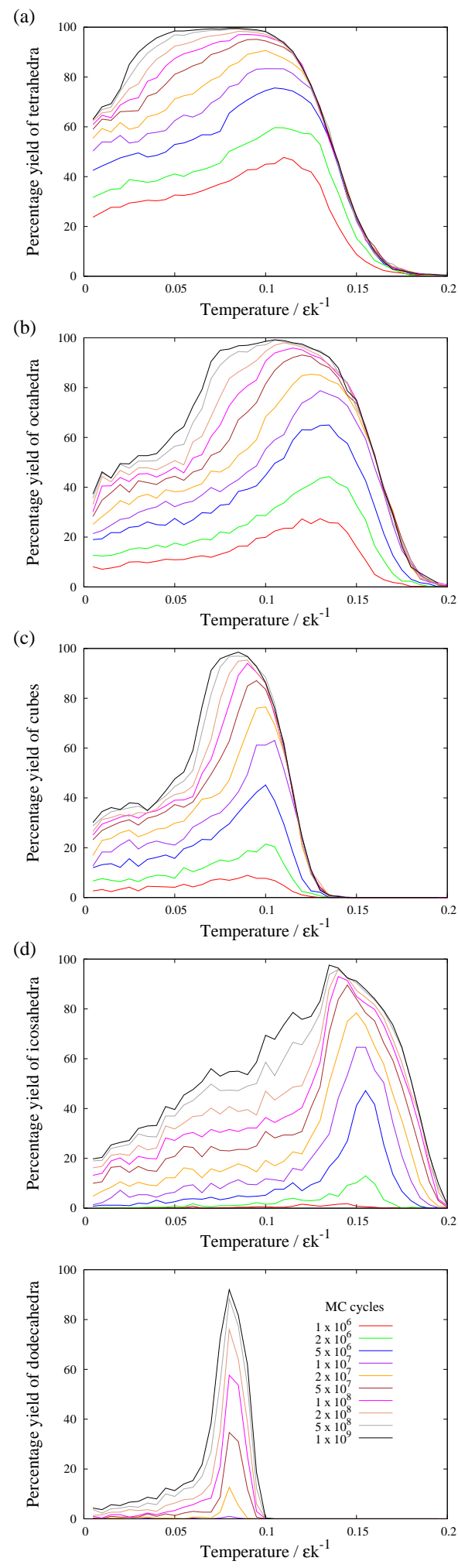

FIG. 6: (Colour Online) The yields of (a) tetrahedra, (b) octahedra, (c) cubes, (d) icosahedra and (e) dodecahedra after different numbers of simulation steps as a function of temperature $T$, in simulations of systems of 1200 particles. In each case the value of $\sigma_{\text {ang }}$ was chosen where maximum yields were obtained in Fig. 1 1 , giving $\sigma_{\text {ang }}=0.5$ except in the case of the dodecahedra, where $\sigma_{\text {ang }}=0.35$. Each data point is an average over five simulations. All the plots use the same key, inset in the plot for dodecahedra.

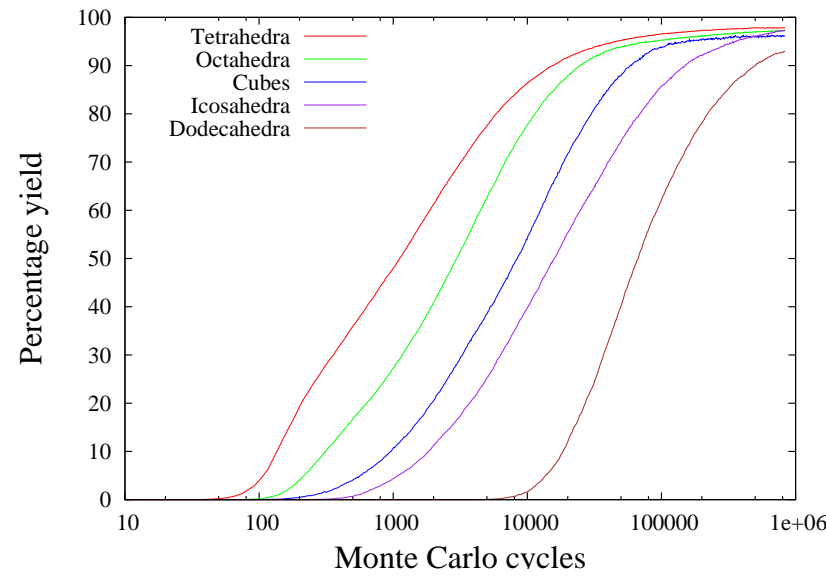

FIG. 7: (Colour Online) The yields of the various target structures as a function of time, under optimal conditions in each case. The temperatures used were as follows. Tetrahedra: $T=0.1$, octahedra: $T=0.12$, cubes: $T=0.09$, icosahedra: $T=0.14$, dodecahedra: $T=0.08 . \sigma_{\text {ang }}=0.5$ for all targets except the dodecahedra, where $\sigma_{\text {ang }}=0.35$. Each line is an average of 100 simulations, each containing 1200 particles.

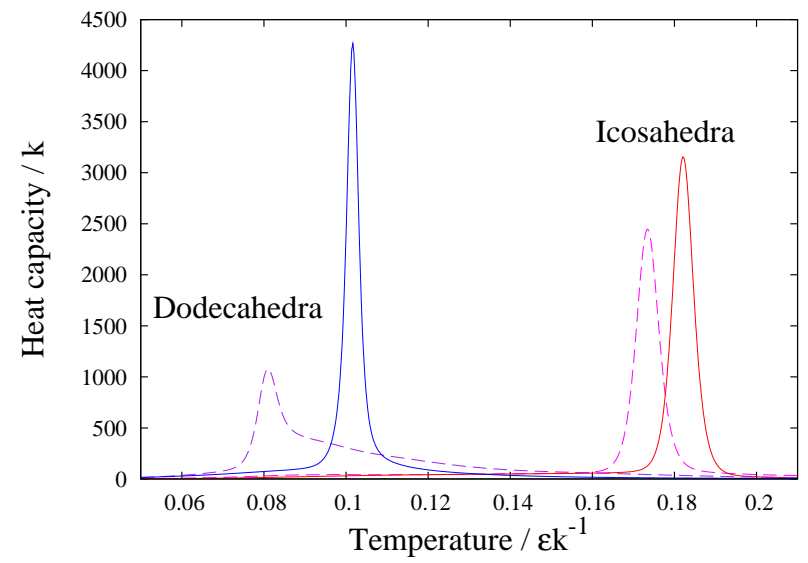

FIG. 8: (Colour Online) The heat capacity $C_{v}$ as a function of temperature for the formation of a single icosahedron and dodecahedron. The dashed lines are the analogous plots for systems with no torsional constraints.

pacity $C_{v}$ as a function of temperature for the formation of a single icosahedron and dodecahedron, with and without torsional constraints. There is a broad shoulder above the main peak of the dodecahedron in the absence of torsional constraints, because, as the temperature is increased, the cluster first melts, before gradually evaporating to form a monomeric vapour. Torsional constraints destabilise the disordered cluster state, leaving a single sharp transition between the dodecahedron and the monomer gas. For the icosahedron no such dramatic change is observed, since the icosahedron directly disassembles into monomers in both cases. Rather, there is just a small increase in $T_{c}$ corresponding to the slight destabilisation of the monomer gas, because it is less non- 
(a)

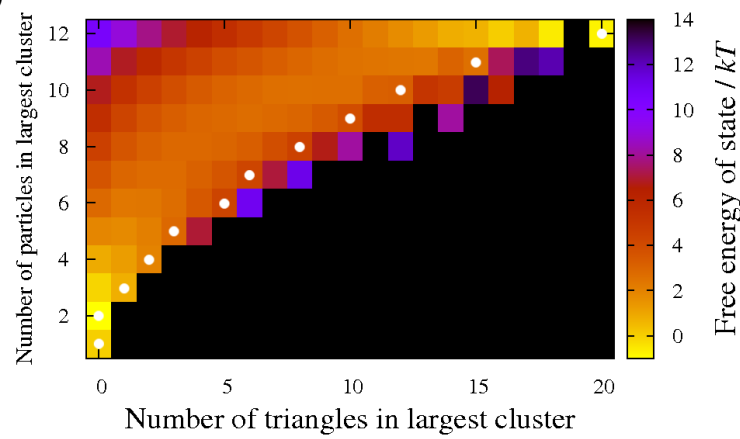

(b)

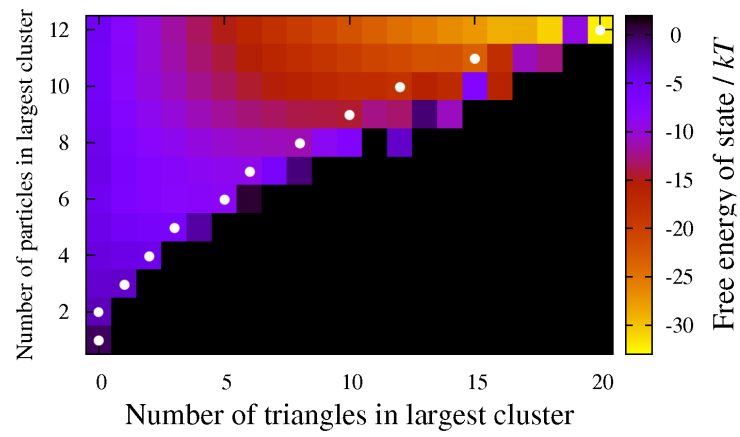

FIG. 9: (Colour Online) Free energy landscapes for the formation of a single icosahedron at $\sigma_{\text {ang }}=0.45$ and (a) $T=0.182$, corresponding to the peak in $C_{v}$, (b) $T=0.16$, the optimal temperature for assembly. The white dots indicate subclusters of the icosahedron that maximize the number of triangles for that clusters size.

ideal.

Two-dimensional free energy landscapes can provide a picture of the pathway for self-assembly. Fig. 9 shows that there is considerable structural order (as measured by the number of triangles) in the intermediates for icosahedron formation. This order is induced by torsional constraints and helps the system avoid kinetic traps associated with misformed clusters and therefore to assemble correctly. At the optimal conditions, the most probable states for each cluster size are those icosahedral intermediates that maximize the number of triangles (Fig. 9(a)). This result provides some support for those kinetic models that just consider the lowest-energy pathway ${ }^{32}$ or a limited set of low-energy pathways. $\frac{36 / 41}{4}$ However, states with fewer triangles still have significant probabilities. Furthermore, at $T_{c}$ the higher entropy of states with fewer triangles makes them more stable, and there is a broad valley across the free energy landscape, thus implying that many pathways are relevant.

Fig. 10(a) shows the same type of free energy landscape for the formation of a dodecahedron at $T_{c}$. Firstly, there are two clear free energy minima with a barrier between them, confirming that the transition is now between a monomeric gas and the assembled dodecahedron. Sec- (a)

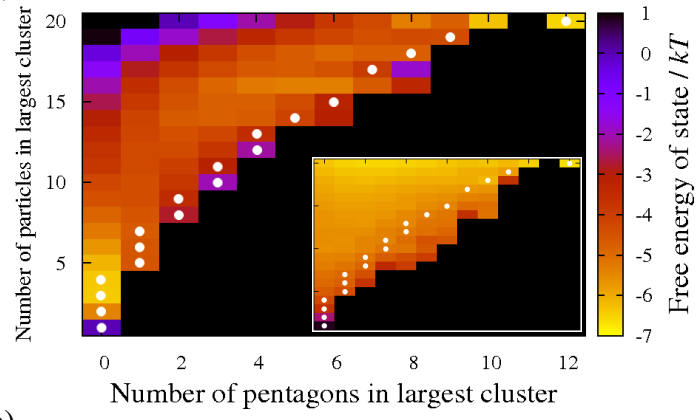

(b)

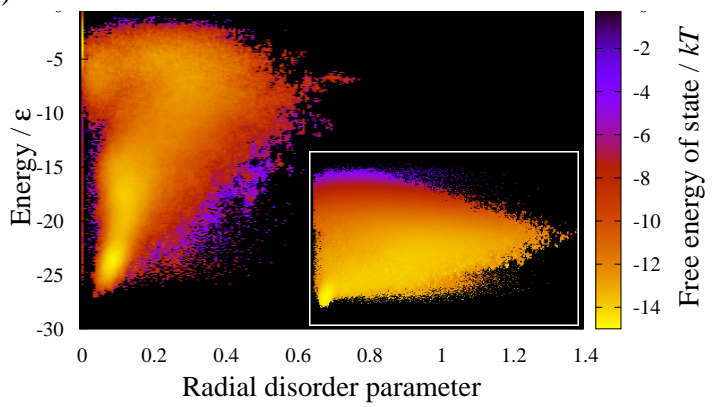

FIG. 10: (Colour Online) Free energy landscapes for the formation of a single dodecahedron. In (a) the order parameters are the numbers of particles and pentagons in the largest cluster, while in (b) they are the potential energy and a measure of radial disorder, described in the text. The insets show the analogous plots for systems with no torsional constraints, where the axes of the insets span the same parameter ranges as the main plots. The white dots in (a) indicate sub-clusters of the dodecahedron that maximize the number of pentagons for that clusters size.

ondly, similar to the icosahedron, there is considerable structural order along the pathway for assembly, and so as clusters grow they are 'guided' towards the dodecahedral target. This free energy landscape contrasts sharply with the analogous landscape for a system without torsional constraints (see inset), for which disordered aggregates are also stable and block the formation of the target structure.

A different view of the free energy landscape is provided in Fig. 10.(b) where the order parameters are the potential energy and a "radial order parameter" that is defined as the standard deviation in the distance of particles from the centre of mass, and takes a value of zero when all particles lie on the surface of a sphere (as in a perfect dodecahedron). In the absence of torsional constraints the system is able to access very disordered states with little energy penalty, and indeed can reach low energies while remaining very far from the dodecahedral structure. Once torsional constraints are included the landscape takes on a classic funnel shape $\frac{\sqrt{66}}{\sqrt{6}}$ such that as the energy of the system decreases it is directed into the dodecahedral structure. 


\section{CONCLUSIONS}

We have extended a patchy particle model that can self-assemble into monodisperse clusters $54 \mid 55$ to include a torsional component in the interparticle potential, better mimicking the nature of protein-protein interactions. We found that similar principles operate in both these models. Firstly, in both cases there is a temperature window over which successful assembly can occur because at too high temperatures the target structure is thermodynamically unstable and at low temperatures the system becomes kinetically trapped. Similarly, there is a limited range of patch specificities associated with successful assembly, because when the patches are too wide alternative configurations become possible, and when the patchpatch interactions are too specific growth is too slow.

However, there are also clear differences between the models. In particular, the torsional constraints greatly reduce the set of erroneous structures that can be formed, and thus remove the competition with large disordered aggregates that was a feature of the original model. So now, rather than large disordered aggregates, the alternative configurations at larger $\sigma$ are malformed shell-like clusters, and at lower temperatures the system becomes trapped in incomplete clusters because of monomer starvation due to the rapid rate of cluster nucleation. Both these features make the behaviour of the model much more similar to that for virus capsids, as would be expected from the more protein-like nature of the interactions.

This lack of competition from aggregates enables the assembly of larger target clusters than was possible in the original model. In particular, the dodecahedron which proved impossible to assemble without torsional interactions readily assembles for the current model. The free energy landscapes for the respective systems dramatically illustrate these differences. With torsional constraints, the free energy landscapes now have a funnel-like topography guiding the system towards the target cluster, and intermediate-sized clusters exhibit a significant amount of the structural order that is characteristic of the target, i.e. as the clusters grow, they grow with the correct structure.

Interestingly, the assembly behaviour for the different targets is very similar, save for the general effects of target size, e.g. the time taken to achieve assembly increases monotonically with size, and the effects of monomer starvation become more pronounced for larger targets. This similarity contrasts with the original non-torsional model, where the dependence of the propensity to aggregate on the patch geometry leads to large differences in behaviour for the different targets. Notably, the ability of all the targets to readily assemble for the current model suggests that proteins are less limited in the geometric forms into which they can assemble, as also seems apparent from the diverse range of biological structures and machines made up of proteins.

If synthetic particles are to begin to mimic this biological repertoire, our results suggest that torsionallyspecific interactions between the particles would be required. However, it is not apparent how such orientational specificity might be achieved for patchy nanoparticles and colloids. By contrast, the DNA units constructed by Mao and coworkers have both 'valency' and control over the relative orientation of the units. Thus, our results help to understand why tetrahedra, cubes, dodecahedra and truncated icosahedra can be successfully assembled from 3-armed units, $\frac{57 / 58}{5}$ and icosahedra from 5-armed units 59 Although far from spherical, the current model may not provide such a bad representation for these DNA systems, especially if internal degrees of freedom for the particles could be added to mimic the flexibility of the arms.

\section{Acknowledgments}

The authors are grateful for financial support from the EPSRC and the Royal Society.
* Author for correspondence

${ }^{1}$ G. M. Whitesides and B. Grzybowski, Science 295, 2418 (2002).

2 D. S. Goodsell, Bionanotechnology (Wiley-Liss, Hoboken, 2004).

3 J. B. Bancroft, G. J. Hills, and R. Markham, Virology 31, 354 (1967).

${ }^{4}$ P. E. Prevelige, D. Thomas, and J. King, Biophys. J. 64, 824 (1993).

5 T. Dokland, Cell. Mol. Life Sci. 56, 580 (1999).

${ }^{6}$ K. N. Parent, S. M. Doyle, E. Anderson, and C. M. Teschke, Virology 340, 33 (2005).

7 K. N. Parent, A. Zlotnick, and C. M. Teschke, J. Mol. Biol. 359, 1097 (2006).

8 D. M. Salunke, D. L. D. Caspar, and R. L. Garcea, Bio- phys. J. 56, 887 (1989).

9 B. Rombaut, R. Vrijsen, and A. Boeyé, Virology 177, 411 (1990).

10 A. Zlotnick, J. M. Johnson, P. W. Wingfield, S. J. Stahl, and D. Endres, Biochemistry 38, 14644 (1999).

11 A. Zlotnick, R. Aldrich, J. M. Johnson, P. Ceres, and M. J. Young, Virology 277, 450 (2000).

12 G. L. Casini, D. Graham, D. Heine, R. L. Garcea, and D. T. Wu, Virology 325, 320 (2004).

13 D. S. Goodsell and A. J. Olson, Annu. Rev. Biophys. Biomol. Struct. 29, 105 (2000).

14 E. D. Levy, J. B. Pereira-Leal, C. Chothia, and S. A. Teichmann, PLoS Comput. Biol. 2, e155 (2006).

15 J. Janin, R. P. Bahadur, and P. Chakrabarti, Q. Rev. Biophys. 41, 133 (2008). 
16 G. C. Ford, P. M. Harrison, D. W. Rice, J. M. A. Smith, A. Treffry, J. L. White, and J. Yariv, Phil. Trans. R. Soc. B 304, 551 (1984).

17 T. Izard, A. Ævarsson, M. D. Allen, A. H. Westphal, R. N. Perham, A. de Kok, and W. G. J. Hol, Proc. Natl. Acad. Sci. USA 16, 1240 (1999).

18 A. Zlotnick and S. J. Stray, Trends Biotechnol. 21, 536 (2003).

19 P. E. Prevelige, Trends Biotechnol. 16, 61 (1998).

20 A. Zlotnick, A. Lee, C. R. Bourne, J. M. Johnson, P. L. Domanico, and S. J. Stray, Nat. Protoc. 2, 490 (2007).

${ }^{21}$ K. Cho, X. Wang, S. Nie, Z. G. Chen, and D. M. Shin, Clin. Cancer Res. 14, 1310 (2008).

22 J. Lewis, G. Destito, A. Zijlstra, M. Gonzalez, J. Quigley, M. Manchester, and H. Stuhlmann, Nat. Med. 12, 354 (2006).

23 S. G. Zhang, Nat. Biotechnol. 21, 1171 (2003).

24 S. C. Glotzer and M. Solomon, Nature Materials 6, 557 (2007).

25 P. K. Sorger, P. G. Stockley, and S. C. Harrison, J. Mol. Biol. 191, 639 (1986).

26 D. Willits, X. Zhao, N. Olson, T. Baker, A. Zlotnick, J. Johnson, T. Douglas, and M. Young, Virology 306, 280 (2003).

27 J. M. Johnson, D. A. Willits, M. J. Young, and A. Zlotnick, J. Mol. Biol. 335, 455 (2004).

${ }^{28}$ K. N. Parent, M. M. Suhanovsky, and C. M. Teschke, J. Mol. Bio. 365, 513 (2007).

29 S. J. Hanslip, N. R. Zaccai, A. P. J. Middelberg, and R. J. Falconer, Biotechnol. Progr. 22, 554 (2006).

${ }^{30}$ C. Bourne, S. Lee, B. Venkataiah, A. Lee, B. Korba, M. G. Finn, and A. Zlotnick, J. Virol. 82, 10262 (2008).

31 R. Tuma, H. Tsuruta, K. H. French, and P. E. Prevelige, J. Mol. Biol. 381, 1395 (2008).

32 D. Endres and A. Zlotnick, Biophys. J. 83, 1217 (2002).

33 A. Zlotnick, J. Mol. Recognit. 18, 479 (2005).

34 D. C. Rapaport, Phys. Rev. E 70, 051905 (2004).

35 D. C. Rapaport, Phys. Rev. Lett. 101, 186101 (2008).

36 D. Endres, M. Miyahara, P. Moisant, and A. Zlotnick, Protein Sci. 14, 1518 (2005).

37 A. Zlotnick, J. Mol. Biol. 366, 14 (2007).

38 R. Zandi, P. van der Schoot, D. Reguera, W. Kegel, and H. Reiss, Biophys. J. 90, 1939 (2006).

39 P. van der Schoot and R. Zandi, Phys. Biol. 4, 296 (2007).

40 R. Twarock, Philos. Trans. R. Soc. London, Ser. A 364, 3357 (2006).

41 T. Zhang and R. Schwartz, Biophys. J. 90, 57 (2006).

42 B. Sweeney, T. Q. Zhang, and R. Schwartz, Biophys. J. 94, 772 (2008).

43 M. F. Hagan and D. Chandler, Biophys. J. 91, 42 (2006).

44 M. F. Hagan, Phys. Rev. E 77, 051904 (2008).

45 O. M. Elrad and M. F. Hagan, Nano Lett. 8, 3850 (2008).

${ }^{46}$ H. D. Nguyen, V. S. Reddy, and C. L. Brooks III, Nano
Lett. 7, 338 (2007).

47 H. D. Nguyen and C. L. Brooks III, Nano Lett. 8, 4574 (2008).

48 H. D. Nguyen, V. S. Reddy, and C. L. Brooks III, J. Am. Chem. Soc. 131, 2606 (2009).

49 D. J. Wales, Philos. Trans. R. Soc. London, Ser. A 363, 357 (2005).

${ }^{50}$ S. N. Fejer, T. R. James, J. Hernandez-Rojas, and D. J. Wales, Phys. Chem. Chem. Phys. 11, 2098 (2009).

51 T. Chen, Z. Zhang, and S. C. Glotzer, P. Natl. Acad. Sci. USA 104, 717 (2007).

52 D. C. Rapaport, J. E. Johnson, and J. Skolnick, Comp. Phys. Comm. 121-122, 232 (1999).

53 P. L. Freddolino, A. S. Arkhipov, S. B. Larson, A. McPherson, and K. Schulten, Structure 14, 437 (2006).

54 A. W. Wilber, J. P. K. Doye, A. A. Louis, E. G. Noya, M. A. Miller, and P. Wong, J. Chem. Phys. 127, 085106 (2007).

55 A. W. Wilber, J. P. K. Doye, and A. A. Louis, J. Chem. Phys. submitted; arXiv.0907.4807.

${ }^{56}$ Y.-S. Cho, G.-R. Yi, S.-H. Kim, S.-J. Jeon, M. T. Elsesser, H. K. Yu, S.-M. Yang, and D. J. Pine, Chem. Mater. 19, 3183 (2007).

57 Y. He, T. Ye, C. Zhang, A. E. Ribbe, W. Jiang, and C. Mao, Nature 452, 198 (2008).

58 C. Zhang, M. Su, Y. He, X. Zhao, P.-A. Fang, A. E. Ribbe, W. Jiang, and C. Mao, J. Am. Chem. Soc. 131, 1413 (2009).

59 C. Zhang, M. Su, Y. He, X. Zhao, P.-A. Fang, A. E. Ribbe, W. Jiang, and C. Mao, Proc. Natl. Acad. Sci. USA 105, 10665 (2008).

60 J. P. K. Doye, A. A. Louis, I.-C. Lin, L. R. Allen, E. G. Noya, A. W. Wilber, H. C. Kok, and R. Lyus, Phys. Chem. Chem. Phys. 9, 2197 (2007).

61 E. G. Noya, C. Vega, J. P. K. Doye, and A. A. Louis, J. Chem. Phys. 127, 054501 (2007).

${ }^{62}$ G. Villar, A. W. Wilber, P. Williamson, A. J.and Thiara, J. P. K. Doye, A. A. Louis, M. N. Jochum, A. C. F. Lewis, and E. D. Levy, Phys. Rev. Lett. 102, 118106 (2009).

${ }^{63}$ E. D. Levy, E. B. Erba, C. V. Robinson, and S. A. Teichmann, Nature 453, 1262 (2008).

64 A. Zlotnick, Virology 315, 269 (2003).

65 A. R. Fersht, Structure and Mechanism in Protein Science (W.H. Freeman \& Co., New York, 1999).

66 J. D. Bryngelson, J. N. Onuchic, N. D. Socci, and P. G. Wolynes, Proteins 21, 167 (1995).

67 It is noteworthy that in the work of Schwartz and coworkers ${ }^{41 \mid 42}$ the final yield is found to increase monotonically with decreasing binding rate constant (analogous to increasing temperature). This unphysical behaviour results from some irreversible steps in their kinetic scheme that means once formed the target structure can never disassemble. 